\title{
Anti- $N$-Methyl-D-Aspartate Receptor Encephalitis in a Young Child With Histological Evidence on Brain Biopsy of Co-existent Herpes Simplex Virus Type 1 Infection
}

Mark A. Ellul MRCP(UK) $)^{1}$, Michael J. Griffiths MRCPCH ${ }^{1,2}$, Anand Iyer MD², Shivaram Avula FRCR ${ }^{2}$, Sylviane Defres $\mathrm{MBChB}^{1}$, Atik Baborie FCRPath ${ }^{3}$, Angela Vincent FRS ${ }^{4}$, Natalie G. Martin $\mathrm{MBChB}^{5}$, Manish Sadarangani DPhil ${ }^{5}$, Andrew J. Pollard FRCPCH PhD 5 , Tom Solomon FRCP PhD ${ }^{1,3}$, Rachel Kneen MRCPCH ${ }^{1,2 *}$

1. Institute of Infection and Global Health, University of Liverpool, Liverpool, UK

2. Alder Hey Children's NHS Foundation Trust, Liverpool, UK

3. The Walton Centre NHS Foundation Trust, Liverpool, UK

4. Nuffield Department of Clinical Neurosciences, John Radcliffe Hospital, Oxford, UK

5. Department of Paediatrics, University of Oxford, and the NIHR Oxford Biomedical Research Centre, Oxford, UK

Keywords: Encephalitis, encephalopathy, autoantibody, $N$-methyl-D-aspartate receptor, herpes simplex virus

Abbreviated title: Anti-NMDAR encephalitis with co-existent HSV infection Running head title: Anti-NMDAR encephalitis and HSV infection

Word count: 1498

Abstract count: 60

Figures: 1

1 supplementary table

References: 10 
*Correspondence to:

Dr. Rachel Kneen

Institute of Infection \& Global Health

Ronald Ross Building

8 West Derby Road

Liverpool

L69 7BE

Email: rachel.kneen@liverpool.ac.uk

\section{Acknowledgements}

We are grateful to the patient and his parents for allowing us to report his case; the laboratory staff at the Pathology Department at the Walton Centre NHS Foundation Trust and to Dr. Anuradha Chawla and the Virology Department at the Royal Liverpool University Hospital for their help with PCR results. We are also grateful to Drs Eileen Baildam, Gavin Cleary, Lisa McCann, Claire Pain, Andrew Riordan, Therese Callaghan and Miss Sasha Burn for their role with clinical management of the case.

\section{Conflicts of interest and source of funding}

This article presents independent work, which received support from the National Institute for Health Research (NIHR) under its Programme Grants for Applied Research Programme (Grant Reference Number RP-PG-0108-10048); the Meningitis Research Foundation (MRF) and the NIHR Oxford Biomedical Research Centre. 
TS is supported by the NIHR Health Protection Research Unit at Liverpool in Emerging and Zoonotic infections at The University of Liverpool.

\section{Potential conflicts of interest}

M.A.E. No conflict

M.J.G. No conflict

A.I. No conflict

S.A. No conflict

S.D. No conflict

A.B. No conflict

A.V. and Oxford University hold patents and receive royalties for antibody tests.

N.G.M. No conflict

M.S. No conflict

A.J.P. No conflict

T.S. No conflict

R.K. No conflict 


\begin{abstract}
We report a 3-year-old boy with anti- $N$-methyl-D-aspartate receptor (NMDAR) encephalitis with a typical syndrome of movement disorder and encephalopathy and evidence of herpes simplex virus (HSV) type 1 infection on brain biopsy. HSV type 1 infection and anti-NMDAR encephalitis are immunologically linked in some cases: this case illustrates that prodromal HSV type-1 infection may be clinically subtle, and easily missed.
\end{abstract}

\title{
INTRODUCTION
}

Anti-NMDAR encephalitis is a recently described autoimmune disorder of the central nervous system (CNS) with a recognizable phenotype consisting of cognitive and psychiatric features, movement disorder and progressively deteriorating encephalopathy. It was first described as a paraneoplastic condition in a series of young women with ovarian teratomas, but more recently has been described in children, who are more likely than adults to have had a prodromal illness before presentation and less likely to have an underlying tumor [1]. The outcome is generally good especially if recognized and treated early with immunosuppressant medication [1]. In this report we describe a child who had anti-NMDAR encephalitis with a classical presentation and a good outcome but also had histological evidence of HSV type 1 infection in neurons.

\section{CASE REPORT}

A previously well 3-year-old Caucasian boy presented with a 2 week change in behavior, irritability and altered sleep pattern followed by difficulty swallowing, drooling, encephalopathy and involuntary limb and facial movements. He had developed a mild fever and coryzal symptoms just before admission. Four weeks earlier he had a mild encephalopathy, consisting of lethargy and excessive sleepiness, with vomiting lasting 3 days. The parents had sought medical attention, and he was given a course of antibiotics for presumed tonsillitis but had apparently made a full recovery. On admission, he was afebrile and encephalopathic with a Glasgow Coma Scale (GCS) of 9 out of 15 with tachycardia, hyperhidrosis and excessive salivation. 
He had evidence of right otitis externa but no other signs of infection. He had a florid movement disorder characterized by orofacial dyskinesia and coarse writhing movements of all 4 limbs. He was treated empirically with intravenous aciclovir (total 10 days) and ceftriaxone (total 7 days). His condition deteriorated rapidly over several hours, with periods of agitation alternating with apneic episodes and reduced conscious level with tachycardia and excessive salivation. He was intubated and transferred to a pediatric intensive care unit.

Initial blood workup was normal and blood cultures were negative. Cerebrospinal fluid (CSF) analysis demonstrated 12 white cells $/ \mathrm{mm}^{3}$ (100\% lymphocytes), protein $58 \mathrm{mg} / \mathrm{dL}$ and glucose $450 \mathrm{mg} / \mathrm{dL}$ with CSF/plasma glucose ratio of 0.7 . CSF microscopy and culture and polymerase chain reaction (PCR) for HSV types 1 and 2, enterovirus, echovirus and parechovirus were negative. Oligoclonal bands were present in serum and CSF with additional bands in CSF, implying a systemic response with an additional CNS restricted response. Magnetic resonance imaging (MRI) of the brain showed evidence of T2 hyperintensity, diffusely involving the right temporal lobe cortex with right hippocampal volume loss, and subtle hyperintensity involving the left amygdala (Figure $1 A-C$ ). Electroencephalography (EEG) demonstrated generalized slowing, most marked over the right cerebral hemisphere, consistent with encephalopathy, without epileptiform features.

A clinical diagnosis of anti-NMDAR encephalitis was made and $500 \mathrm{mg}$ of intravenous methylprednisolone was given once daily on days 5-7 of admission followed by intravenous immunoglobulin (IVIg): total dose $2 \mathrm{~g} / \mathrm{Kg}$. He was extubated after 5 days, but his conscious level continued to be reduced and the florid movement disorder continued.

Given the unusual MRI findings, which were consistent with HSV type 1 encephalitis, lesional brain and meningeal biopsies were performed on day 10 after admission (Figure $1 E-I$ ). These showed a non-specific active chronic inflammation with occasional polymorphonuclear cells, multinucleated giant cells and plasma cells, scattered macrophages and lymphocytes in meninges, white and cortical type grey matter consistent with meningoencephalitis. The brain parenchyma had a positive PCR for HSV type 1, confirmed on repeat testing, and immunohistochemistry for 
HSV type 1 showed occasional very faint cytoplasmic staining in neuronal-type cells (Figure 1I). Aciclovir was recommenced and given intravenously for a further 21 days. Anti-NMDAR antibodies ( $\mathrm{IgG}$ ) were subsequently reported to be highly positive in serum and CSF. Voltage-gated potassium channel (VGKC) complex antibodies, glutamic acid decarboxylase (GAD) antibodies and a panel of onconeuronal antibodies were negative. Plasma exchange was commenced on day 12; he received 15 single volume cycles without complications. On day 15, CSF demonstrated 2 white cells $/ \mathrm{mm}^{3}$, PCR for HSV type 1 was negative and anti-NMDAR antibodies were negative. Anti-NMDAR serum antibodies were still present on day 15 at low levels but were negative at day 33 .

Given the severity of his encephalitis, he took a 6 week tapering course of oral prednisolone, infusions of cyclophosphamide every month for 6 months (cumulative dose of $\left.2250 \mathrm{mg} / \mathrm{m}^{2}\right)$ and 2 rituximab cycles, given 6 months apart $\left(1500 \mathrm{mg} / \mathrm{kg} / \mathrm{m}^{2}\right.$ given on 2 consecutive days, 2 weeks apart). A repeat MRI 8 months after presentation revealed atrophy of the right medial temporal lobe with bilateral cerebral parenchymal volume loss and post-biopsy subdural effusions (Figure $1 D$ ). He was discharged after 4 months of neurorehabilitation having made a good recovery. At last follow-up, 24 months after presentation, he had no residual physical neurological disability. Formal neuropsychology has not been performed due to his young age but the parents report that his understanding and expression of speech, sleep and behavior are normal. Anti-NMDAR antibodies in serum remain negative.

\section{DISCUSSION}

There is increasing evidence of an association between HSV type 1 encephalitis and the presence of anti-NMDAR antibodies. To date, 33 cases of HSV type 1 encephalitis in adults $(n=18)$ and children $(n=15)$ have been reported in close temporal relationship to the identification of anti-NMDAR antibodies in the serum and/or CSF [2-9] (Supplementary Table 1).

Several small case series and case reports have identified patients, primarily children, with clinical relapse following proven HSV type 1 encephalitis. These relapses were associated with development of movement disorders, not seen during the HSV 
encephalitis, and anti-NMDAR antibodies in the serum, and in CSF when available [3-9]. In 7 cases, sera and CSF from the original HSV encephalitis period were shown to be negative for anti-NMDAR antibodies, demonstrating that these developed only during relapse $[5,6,7,9]$.

In another retrospective study, stored acute serum from a cohort of 44 adults diagnosed with HSV type 1 encephalitis (CSF PCR positive), were tested for antiNMDAR antibodies [2]. None had clinical features suggestive of anti-NMDAR encephalitis, but 13 had anti-NMDAR antibodies with a mixture of $\operatorname{IgA}$, IgM and IgG classes. All the anti-NMDAR antibody positive patients had extensive temporal lobe hyperintensity on MRI imaging in keeping with the typical appearances of HSV type 1 encephalitis, and additional imaging features were not reported.

Our patient differs from those in previous reports as he did not have features of severe clinical HSV type 1 encephalitis initially. Also, the CSF PCR for HSV type 1 was negative throughout the time of hospitalisation, although no lumbar puncture was undertaken during his initial mild encephalopathy. Nevertheless, PCR of the parenchyma of the temporal lobe lesion was positive for HSV type 1, 25 days from onset of symptoms and there was histological evidence of infection within some of the neurons. Given his age, it seems likely that this was a primary infection, possibly occurring during his earlier mild encephalopathy.

The temporal lobe changes seen on the MRI, including hippocampal volume loss, are also consistent with a previous HSV infection with residual damage. MRI findings in anti-NMDAR encephalitis are usually less florid (or normal) and include abnormalities in the medial temporal lobes or less frequently in the thalamus or occipital cortex [10]. Therefore, the evidence of bilateral medial temporal lobe involvement in addition to right-sided diffuse temporal lobe involvement in this case is an important finding that supports features of both HSV type 1 and anti-NMDAR encephalitis.

Our patient had a mild and brief encephalopathy 4 weeks prior to presentation; we therefore hypothesize that he had mild HSV type 1 encephalitis at this point but by admission he was displaying a secondary anti-NMDAR encephalitis syndrome. The 
clinical features of HSV type 1 infection may have been less severe because the nondominant temporal lobe was most affected. The response to immunotherapy observed, and the correlation between the fall in anti-NMDAR antibody titres and clinical improvement, suggest that the pathological process at the time of presentation was primarily antibody mediated rather than as a result of viral infection.

The mechanism by which viral infection leads to the production of autoantibodies is not clear. Inflammation and viral neuronal lysis caused by HSV type 1 infection may result in the exposure of neuronal surface proteins, leading to antibody production directed against antigens to which tolerance is normally preserved [7]. Alternatively, a process of molecular mimicry between virus-associated antigens and the NMDA receptor may occur [2]. However, the existence of other infections co-incident with anti-NMDAR encephalitis, such as adenovirus identified in the CSF of one child, argues against specific molecular mimicry involving HSV type 1 [3]. Further studies are needed to characterize the role of anti-NMDAR antibodies in HSV type 1 encephalitis, as well as to establish the prevalence of active infection with HSV type 1 or other viruses in patients with anti-NMDAR encephalitis.

Our findings suggest that some patients with anti-NMDAR encephalitis may have had a preceding subtle HSV type 1 infection which may be the precipitant for an autoimmune process, and that more cases of anti-NMDAR encephalitis may be associated with HSV type 1 than currently recognised using CSF PCR only. 


\section{References}

1. Florance NR, Davis RL, Lam C, et al. Anti-N-methyl-D-aspartate receptor (NMDAR) encephalitis in children and adolescents. Ann Neurol. 2009;66(1):11-8.

2. Prüss H, Finke C, Höltje M, et al. N-methyl-D-aspartate receptor antibodies in herpes simplex encephalitis. Ann Neurol. 2012;72(6):902-11.

3. Hacohen Y, Wright S, Waters P, et al. Paediatric autoimmune encephalopathies: clinical features, laboratory investigations and outcomes in patients with or without antibodies to known central nervous system autoantigens. J Neurol Neurosurg Psychiatry. 2013;84(7):748-55.

4. Mohammad SS, Sinclair K, Pillai S, et al. Herpes simplex encephalitis relapse with chorea is associated with autoantibodies to N-Methyl-D-aspartate receptor or dopamine-2 receptor. Mov Disord. 2014;29(1):117-22.

5. Hacohen Y, Deiva K, Pettingill P, et al. N-methyl-D-aspartate receptor antibodies in post-herpes simplex virus encephalitis neurological relapse. Mov Disord. 2014;29(1):90-6.

6. Hacohen $\mathrm{Y}$, Absoud M, Hemingway C, et al. NMDA receptor antibodies associated with distinct white matter syndromes. Neurol Neuroimmunol Neuroinflamm. 2014;24;1(1):e2.

7. Armangue T, Leypoldt F, Málaga I, et al. Herpes Simplex Virus Encephalitis is a Trigger of Brain Autoimmunity. Ann Neurol. 2014;75(2):317-23.

8. Desena A, Graves D, Warnack W, Greenberg BM. Herpes Simplex Encephalitis as a Potential Cause of Anti-N-Methyl-d-Aspartate Receptor Antibody Encephalitis: Report of 2 Cases. JAMA Neurol. 2014;71(3):344-6. 
9. Wickström R, Fowler A, Cooray G, Karlsson-Parra A, Grillner P. Viral triggering of anti-NMDA receptor encephalitis in a child - An important cause for disease relapse. Eur J Paediatr Neurol. 2014;18(4):543-6.

10. Baumgartner A, Rauer S, Mader I, Meyer PT. Cerebral FDG-PET and MRI findings in autoimmune limbic encephalitis: correlation with autoantibody types. $J$ Neurol. 2013;260(11):2744-53. 


\section{FIGURE LEGENDS}

\section{Figure 1.}

$A-C$, T2 weighted coronal $(A, B)$ and axial $(C)$ MRI images from 3-year-old child taken on day 3 after presentation with anti-NMDAR encephalitis showing T2 hyperintensity involving the right temporal lobe cortex (white arrows) including the hippocampus which demonstrates volume loss (open arrow). Focal T2 hyperintensity is seen within the left amygdala (black arrows). $D$, coronal MRI image from 8 months after presentation showing atrophy of the right temporal lobe (arrow) with bilateral cerebral parenchymal volume loss and associated subdural effusions. The larger right sided subdural effusion is evident (arrow heads).

$E-I$, images of meningeal biopsy $(E-F)$ and brain biopsy $(G-H)$ from day 10 after presentation, E-H, stained with hematoxylin and eosin, showing active chronic inflammation with an occasional multinucleated giant cell. I, HSV type 1 immunohistochemistry (Dako HSV1 antibody B0114, dilution 1:2000) showing an occasional neuronal type cell with very faint cytoplasmic staining. Bars represent 100 $\mu \mathrm{m}$ for $E$ and $G ; 20 \mu \mathrm{m}$ for $F, H$ and $I$.

\section{Supplementary Table 1.}

Review of 33 patients with HSV type 1 encephalitis (PCR positive in CSF) with associated anti-NMDAR serum antibodies.

D, days; Mo, months; Y, years; M, male; F, female; NMDAR, $N$-methyl-D-aspartate receptor; CSF, cerebrospinal fluid; N/A, not available; HSV, herpes simplex virus; MP, methylprednisolone; IVIg, intravenous immunoglobulin; Cycloph, cyclophosphamide; Cyclosp, cyclosporine; PLEX, plasma exchange; MMF, mycophenolate mofetil; Ritux, rituximab; Pred, prednisolone. 